\title{
3 Dimensional Motion of Photon and Its Energy
}

\author{
Gh. Saleh ${ }^{1, *}$, Reza Alizadeh ${ }^{1}$, Ehsan Dalili ${ }^{1}$, and Amir Noorbakhsh ${ }^{1}$ \\ ${ }^{1}$ Saleh Research Centre, Netherlands
}

\begin{abstract}
Since the electron is the photon-generator and the electron is rotating around itself and around the nucleus, the emitted photon from the electron should have the effect of these rotating motions. So, the photon has a three-dimensional motion, including a transition movement and a rotary motion. And it traverses in a helical trajectory. By using this definition, we have proved wave-particle duality at the same time and introduce a new equation for the photon motion and its energy.
\end{abstract}

\section{Introduction}

For a long time, the smallest object in the universe, which now is called an atom, was unknown. Until recently (a hundred years ago), no structure was imagined for that, but its structure is clear now as it could be fission. Like the atom, the photon has a world that is like an old atom for us, unfamiliar and anonymous but at the same time obvious. The photon is a child of the 20th century. In 1926, the photon so named by the physical chemist Gilbert Nor-ton Lewis. It is the 'particle of light' -or 'light quantum' - first hypothesized by Albert Einstein in 1905, and then used by him to explain the photoelectric effect.[1-3]

Saleh Research Group aims that with a new and fresh approach and with very strong reasons, reveal the unexplored depths and strengthen its connection with the beautiful photon. In this article, the behavior of photon is studied in three dimensions (instead of 2) [4]. And we present a new definition for photon's motion and new formula for its energy.

\section{The photon genesis}

We return to the beginning of creation of a photon from the heart of an atom, from its mother's place, which is the electron. At $\mathrm{T}=0+\varepsilon$, as the electron is excited, or in other words, absorbed the given energy, it loses the extra energy or come back to the ground state by emitting an object called "photon"[5]. An important point to note is that electron rotates around itself and around its nucleus at the very high speed. So when a photon is released, it will continue to move at the speed of light and because of the very fast rotational motion of electron, it has a rotational motion too. In an obvious matter, an emitted photon from an electron has a linear and rotational motion. In fact, our rotating photon moves in at least three dimensions.

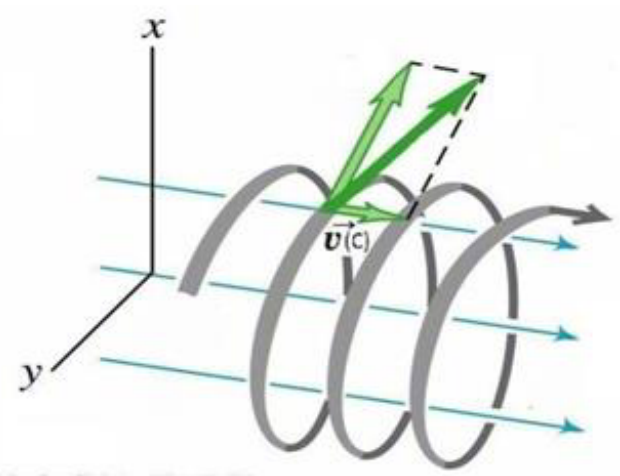

Fig. 1. 3 dimensional motion of photon [4]

\section{The energy of Rotational photon}

It should be noted that when a photon is emitted from an electron, it is like a spaceship, like a bullet, that has been given primary energy and thrown into space. Inevitably, when you observe it from the front, you will find it in one form and when you observe it from the side view, you will find it in the other form.[4] The front view of a photon is the image of a helical motion toward an observer, a circle with a radius of $\mathrm{R}_{\text {mom }}$, and the side view of the moving photon is a sinusoidal motion.

On the other hand the equation of motion and energy of photon must show this behavior too. In fact, the emitted photon has an initial energy from which must be subtracted the Rotational kinetic energy to have the sum of the Translational kinetic energy:

\section{Translational kinetic energy $=$ \\ Initial energy - Rotational kinetic energy}

Saleh Theory knows the Translational kinetic energy as the same energy that Thomas Planck had presented with his famous formula and measured empirically in the laboratory: 


$$
\mathrm{E}=\mathrm{h} \vartheta
$$

The initial energy is:

$$
\mathrm{E}_{\mathrm{i}}=1 / 2 \mathrm{mv}^{2}
$$

The rotational energy is:

$$
\mathrm{E}_{\mathrm{r}}=1 / 2 \mathrm{mr}^{2} \omega^{2}
$$

So:

$$
\mathrm{E}=\mathrm{h} \vartheta=1 / 2 m v^{2}-1 / 2 \mathrm{mr}^{2} \omega^{2}
$$

This new formula has three different parts which one part is the energy of wave-like part, and the second one is particle part, and the third part refers to the rotating energy that the color variety of the photon is related to the third part.

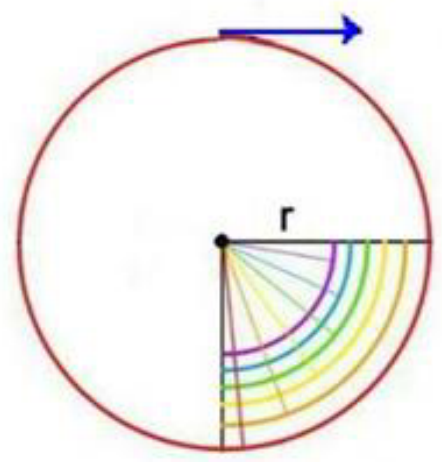

Fig. 2. Front view of photon motion of photon [4]

\section{Results and Discussion}

Today's all theories explain photon's motion separately. Sometimes they knew photon like wave (double-slit experiment, etc.) and sometimes like particles (photoelectric effect, etc.). But when we focused on the behavior of the generator of photon, electron, we have found that photon should have 3 dimensional motion not 2 [4]. So we presented this new motion for photon and regarding that the energy formula for that.

\section{References}

Here are some examples:

1. N. Mukunda, "The story of the photon." Resonance 5.3 (2000).

2. Pais, Abraham. Subtle is the Lord: The Science and the Life of Albert Einstein: The Science and the Life of Albert Einstein. Oxford University Press, USA, 1982.

3. J.J. Stachel,. "Einstein's miraculous year: five papers that changed the face of physics." (2005).
4. Gh. Saleh, et al. "A New Explanation for the Color Variety of Photons." MATEC Web of Conferences. Vol. 186. EDP Sciences,( 2018).

5. Gh. Saleh, et al. "The Superstring Theory and the Shape of Protons and Electrons." MATTER: International Journal of Science and Technology 4.2 (2018) 